\title{
Three-dimensional Porous Graphene/Polyaniline Hybrids for High Performance Supercapacitor Electrodes
}

\section{Zhaoxia HOU *, Peng SHI, Shengnan ZOU}

Liaoning Province Key Laboratory of Micro-nano Materials Research and Development, School of Mechanical Engineering, Shenyang University, Shenyang 110044, China

*Corresponding Author: Zhaoxia HOU, School of Mechenical Engineering, Shenyang University, Shengyang, 110044, P. R. China E-mail address:Iuckyxia2007@126.com

\begin{abstract}
:
Graphene-based composites took extensive attraction as electrodes for supercacitors these years. Three-dimensional cross-linking porous graphene (3D rGO-m) was obtained by $\mathrm{KOH}$ activation to graphene modified by 1,2,4-triaminobenzene. 3D porous graphene/ polyaniline hybrids (3D rGO-m/PANI) was prepared by the in-situ chemical oxidative polymerization. The rGO-m are reconstructed from $2 \mathrm{D}$ to $3 \mathrm{D}$ porous structure after $\mathrm{KOH}$ activation. The PANI nanorod arrays are successfully decorated on the surface of the $3 \mathrm{D}$ porous graphene sheets. The specific capacitance of the $3 \mathrm{D} \mathrm{rGO}-\mathrm{m} / \mathrm{PANI}$ hybrids reach $985 \mathrm{~F} / \mathrm{g}$ at $0.5 \mathrm{~A} / \mathrm{g}$. The capacitance retention of 3D rGO-m/PANI maintains $90 \%$ of its initial capacity after 1000 cycles, while rGO-m/PANI only keeps $83 \%$ of its initial capacity, the cycling stability of both hybrids are higher than that of pure PANI (69\%).
\end{abstract}

Keywords: three dimension; porous graphene; polyaniline; hybrid materials; supercapacitor

\section{Introduction}

Compared to conventional capacitors and $\mathrm{Li}$ ion batteries, supercapacitors with outstanding charge/ discharge rate and high power capability have attracted considerable research, and will become a promising energy storage device ${ }^{[1]}$. Electrodes, as the key materials in energy storage devices, determine the performance of the supercapacitors, such as capability, delivery rates and cycling stability ${ }^{[2]}$.

Among carbon based materials, such as active carbon, carbon nanotube, graphene is a highly promising electrode material in Electric double layer capacitor (EDLC) due to its high electrical conductivity, high specific surface area, high charge mobility, favorable flexibility, and good mechanical strength ${ }^{[3]}$. However, graphene sheets are easy to aggregate due to the strong $\pi-\pi$ interaction. The aggregation of graphene makes the channels among the sheets so narrow that the electrolyte ions are unable to access ${ }^{[4]}$. Compared to $2 \mathrm{D}$ graphene sheets, 3D porous graphene structure can provide larger specific surface area, which will contribute larger contact interfaces between electrode and electrolyte, and provide three-dimensional paths for ionic or electronic transport as well, so $3 \mathrm{D}$ porous structure graphene is considered to have excellent electrochemical performance.

Polyaniline (PANI) with low cost, easy synthesis and high pseudocapacitance has become a promising pseudo supercapacitor electrode material. PANI with highlyordered nanowire arrays are beneficial to facilitate ion transport by shorten transport paths ${ }^{[5]}$. However, the inevitable volume expansion and contraction during change/discharge will result in mechanical degradation and low cycling stability, which constraint its practical application ${ }^{[6]}$. So, it is put forward that combining PANI with graphene or other inorganic materials such as metals or metal oxides as scaffold or bracket to confine the volume changes of PANI, hybrid materials will possess both high capacitance and good cycling stability ${ }^{[7-11]}$.

In this work, we proposed an effective method to prepare $3 \mathrm{D}$ porous graphene/PANI hybrid materials by $\mathrm{KOH}$ activation and in-situ chemical oxidative polymerization methods. Graphene was modified by 1,2,4-triaminobenzene, the modifier serves as molecular spacer that decreases greatly the stacking of the graphene sheets. Graphene modified (rGO-m) is of thin sheets and crumpled morphology. $\mathrm{KOH}$ activation was used to etch pores on the rGO-m sheets to construct $3 \mathrm{D}$ porous structural graphene ( $3 \mathrm{D} \mathrm{rGO}-\mathrm{m})$, and then $3 \mathrm{D}$ porous 
structure $\mathrm{rGO}-\mathrm{m} / \mathrm{PANI}$ hybrid materials was obtained by in-situ polymerization. The microstructure, morphology and electrochemical performance of the $3 \mathrm{D} \mathrm{rGO}-\mathrm{m} / \mathrm{PANI}$ hybrid materials were characterized and investigated. The 3D rGO-m/PANI hybrid materials showed high specific capacitance and good rate capability and cycling stability.

\section{Experimental}

\subsection{Materials}

Graphite powder $(30 \mu \mathrm{m})$ was from Nanjing XFNANO Materials Tech Co., Ltd. Aniline (An, AR), ammonium persulfate (APS, AR) were from Tianjin Damao Chemical Agent Company. Sodium carbonate anhydrous (Na2CO3, $\mathrm{AR}$ ) and potassium hydroxide ( $\mathrm{KOH}, \mathrm{AR})$ were from Tianjin Bodi Chemical Engineering Corporation. 1,2,4-triaminobenzene dihydrochloride (96\%) was from Alfa Aesar. All reagents were used directly without any treatment.

\subsection{Preparation of $3 \mathrm{D}$ porous $\mathrm{rGO}-\mathrm{m} / \mathrm{PANI}$ hybrids}

2.2a 3D rGO-m: Graphene oxide (GO) was prepared by a modified Hummers method according to the reference ${ }^{[12]}$. Graphene modified by 1,2,4- triaminobenzene (rGO-m) was prepared according to the reference ${ }^{[13]}$. rGO-m were soaked in aqueous $\mathrm{KOH}$ solution with the mass ratio of KOH:rGO-m $=7$ for $24 \mathrm{~h}$, and then heat treated at $500{ }^{\circ} \mathrm{C}$ for $5 \mathrm{~h}$. After activation, samples were sequentially washed by acetic acid and deionized water and further dried at $60{ }^{\circ} \mathrm{C}$ for $12 \mathrm{~h}$. Samples obtained are named as 3D rGO-m. In comparison, the rGO was synthesized via the same procedure as rGO-m but without 1,2,4-triaminobenzene.

$2.2 \mathrm{~b} 3 \mathrm{D}$ porous $\mathrm{rGO}-\mathrm{m} / \mathrm{PANI}$ hybrids: The $3 \mathrm{D}$ rGO-m/PANI hybrids were synthesized by in-situ chemical oxidative polymerization method with aniline as a monomer in the presence of $3 \mathrm{D}$ rGO-m. The procedures were as followed: $3 \mathrm{D}$ rGO-m was ultrasonically dispersed in $1 \mathrm{M} \mathrm{HCl}$ for $0.5 \mathrm{~h}$, and aniline and polyving akohol with 1:0.000075 molar ration were added into 3D rGO-m suspension and performed another $0.5 \mathrm{~h}$ sonication and then decreased the temperature to $0{ }^{\circ} \mathrm{C}$. A certain amount of APS was dropped in and kept stirring at $0{ }^{\circ} \mathrm{C}$ for $8 \mathrm{~h}$. The hybrid materials were filtered and washed repetitively with DI water and ethanol, until the filtrate became colorless. In order to comparison, pure PANI and inactivated rGO-m/PANI hybrids (marked as rGO-m/PANI) were synthesized, respectively, via the same procedures as reported above.

\subsection{Materials Characterization}

The electrodes were prepared by active materials with $10 \%$ binder (polytetrafluoroethylene, PTFE). The two-electrod system was assembled with a cellulose paper as separator, carbon fibre paper as current collector and $1 \mathrm{M} \mathrm{H}_{2} \mathrm{SO}_{4}$ aqueous as electrolyte.

X-ray diffraction (XRD, PANalytical) was analyzed with $\mathrm{Cu} \mathrm{Ka}$ radiation $(\lambda=0.15418 \mathrm{~nm})$ from $5^{\circ}$ to $60^{\circ}$. Foutier transform infrared spectrometer (FT-IR, Bruker EQUNOX 55) was recorded at room temperature from
$400 \mathrm{~cm}^{-1}$ to $4000 \mathrm{~cm}^{-1}$ at a resolution of $2 \mathrm{~cm}^{-1}, \mathrm{KBr}$ powder pellet. The microstructure was observed by the Field Emission Scanning Electron Microscope (FE-SEM, Hitachi S4800).

All the electrochemical performance was studied on a PARSTART 2237 electrochemical workstation. Cyclic voltammetry (CV) and electrochemical impedance spectroscopy (EIS) and galvanostatic charge-discharge (GCD) measurments were carried out in a two-electrode system. $\mathrm{CV}$ and GCD measurements were conducted within the potential range of $0-0.8 \mathrm{~V}$. CV curves were tested at a scan rate of 5, 10, 25, 50 and $100 \mathrm{mV} / \mathrm{s}$. GCD curves were set in the range from $0.01 \mathrm{~Hz}-100 \mathrm{kHz}$ at different current densities.

The specific capacitance (Cs) of the active electrode material was determined from the GCD curves using the equation:

$C_{S}=\frac{4 I \Delta t}{m \Delta V}$

Where, I is discharge current $(\mathrm{A}), \Delta \mathrm{t}$ is the discharging time (s), $\mathrm{m}$ is the total mass of active electrode material (g), and $\Delta \mathrm{V}$ is the voltage drop $(\mathrm{V})$ upon discharging (excluding the IR drop). The energy density E was calculated by

$E=\frac{C_{S} \cdot \Delta V^{2}}{8}$

where the specific capacitance (Cs) was derived from GCD measurement. The same $\Delta \mathrm{V}$ was used as that in Cs calculation from GCD. The power density $\mathrm{P}$ was calculated by

$P=\frac{E}{\Delta t}$

where $\mathrm{E}$ referred to the energy density, $\Delta \mathrm{t}$ was the same as that in calculation of $\mathrm{E}$.

\section{Results and Discussion}

\subsection{Microstructure and morphology}

After a hydrothermal reaction, rGO without modified appears flat and inter-stacked sheets under a FE-SEM (figure 1(a)).The rGO-m appears crumpled sheets (figure 1(b)). Some sheets are translucent, indicating that their thickness in the low side. The rGO-m with crumpled morphology suggests that the amino groups of $1,2,4$ triaminobenzene are chemically bonded with grapheme, which results in the morphology change of the rGO-m sheets. Typical FE-SEM images of activated graphene samples of $3 \mathrm{D}$ rGO-m are shown in figure $1(\mathrm{c})$, it can be seen clearly that the sample activated by $\mathrm{KOH}$ is reconstructed from $2 \mathrm{D}$ to $3 \mathrm{D}$ structure, and highly porous morphology can be observed as well. Restructuring among carbon fragments is due to the dangling bonds resulted from $\mathrm{KOH}$ activation. In the activation process, the etching of $\mathrm{rGO}-\mathrm{m}$ matrix via redox reaction between $\mathrm{C}$ and $\mathrm{KOH}$ leaves defects and planar pores, and produces graphene fragments with a lot of $\mathrm{C}$ dangling bonds. Thus, the restructure of graphene fragments occurs.

Pure PANI is random relatively long rod with $400 \mathrm{~nm}$ 
in length and $60 \mathrm{~nm}$ in diameter (figure 2(a)). The FE-SEM images of rGO-m/PANI shows the structure of rGO-m supported PANI nanorod arrays with $100 \mathrm{~nm}$ in length and $50 \mathrm{~nm}$ in diameter (figure 2(b)), and the rGO-m sheets are obviously visible. 3D rGO-m/PANI shows the similar structure with rGO-m supported PANI nanorod arrays, but owing to the 3D cross-linking structure of rGO-m after $\mathrm{KOH}$ activation, the hybrids of $\mathrm{rGO}-\mathrm{m} / \mathrm{PANI}$ is also cross-linked (figure 2(c)), the morphology of nanorod array and cross-linking sheets is favor for the fast ion or electron transport and expected to increase the specific capacitance, which will be illustrates later.
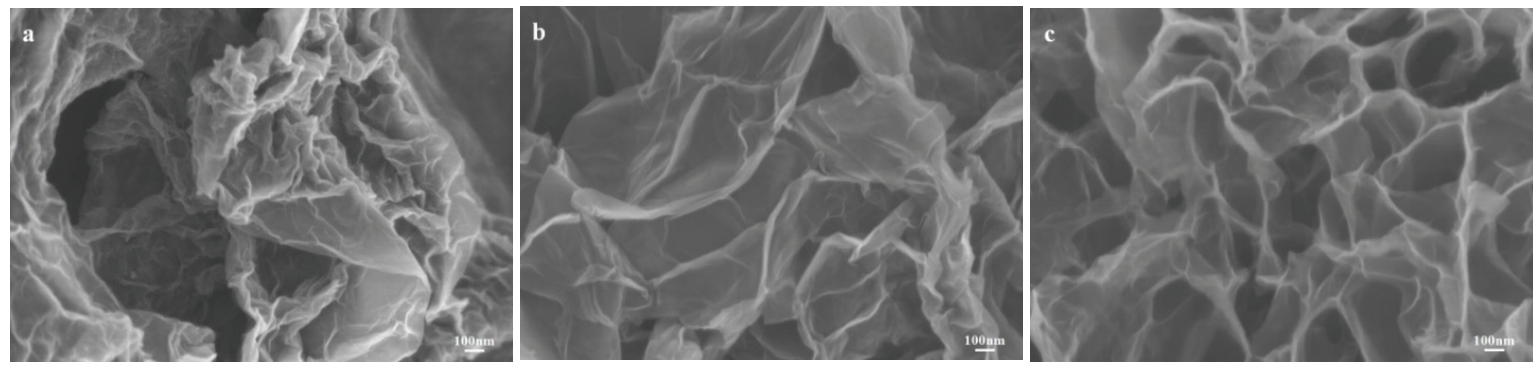

Figure 1. SEM images of (a) rGO, (b) rGO-m and (c) 3D rGO-m.
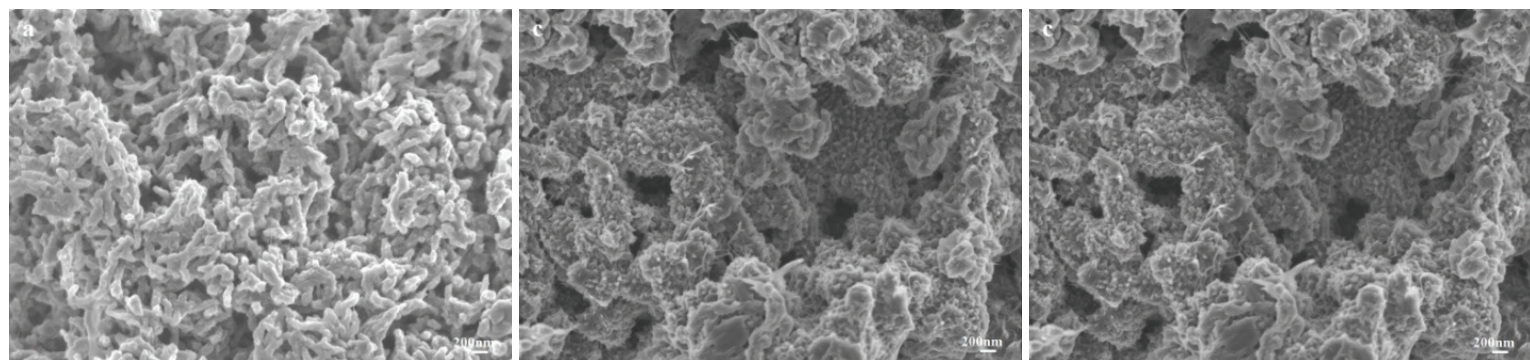

Figure 2. SEM images of (a) PANI, (b) rGO-m/PANI and (c) 3D rGO-m/PANI.

Figure 3 represents the XRD patterns of $\mathrm{GO}, \mathrm{rGO}-\mathrm{m}$, PANI, rGO-m/PANI and 3D-rGO-m/PANI. A broad and intense peak appears at $2 \theta=11.0^{\circ}$ is attributed to the (001) plane basal diffraction of $\mathrm{GO}$, with an inter-layer spacing of $0.80 \mathrm{~nm}$ in the layer-like GO sheets. After modification, the major peak of rGO-m is shifted to $26.4^{\circ}(0.34 \mathrm{~nm})$, it is indicated that $\mathrm{rGO}-\mathrm{m}$ has poor stacking order, and some single layer rGO were formed by completely exfoliation, and fewer layer rGO and/or even loosely stacked layers exist as well ${ }^{[14]}$.

As for rGO-m/PANI and 3D rGO/PANI hybrids, four broad peaks centered at $2 \theta=9.6^{\circ}, 15.1^{\circ}, 20.3^{\circ}$ and $25.2^{\circ}$ can be observed, which are corresponded to (001), (011), (020) and (200) planes of PANI in an emerraldine salt form, respectively ${ }^{[15]}$. XRD patterns of rGO-m /PANI and $3 \mathrm{D}$ rGO/PANI hybrids are similar with PANI, and the peaks of $\mathrm{rGO}$ at around $26.4^{\circ}$ and around $43.1^{\circ}$ still can be observed in rGO-m /PANI and 3D rGO/PANI hybrids. This indicates that $\mathrm{rGO}$ is fully contacted with PANI and PANI is successfully coated on the rGO sheets with $\pi$-stacking between themselves ${ }^{[16]}$.

Figure 4 gives the FT-IR spectra of GO, rGO, rGO-m, PANI, rGO-m/PANI and 3D-rGO-m/PANI. The spectrum of $\mathrm{GO}$ shows the absorption peak of carbonyl $\mathrm{C}=\mathrm{O}\left(v_{\mathrm{C}=\mathrm{O}}\right.$ at $\left.1722 \mathrm{~cm}^{-1}\right)$, benzenoid $\mathrm{C}=\mathrm{C}\left(v_{\mathrm{C}=\mathrm{C}}\right.$ at $\left.1574 \mathrm{~cm}^{-1}\right)$, epoxy $\mathrm{C}-\mathrm{O}-\mathrm{C}\left(v_{\mathrm{C}-\mathrm{O}-\mathrm{C}}\right.$ at $\left.1044 \mathrm{~cm}^{-1}\right)$, hydroxyl C-OH $\left(v_{\mathrm{C}-\mathrm{OH}}\right.$ at $\left.1224 \mathrm{~cm}^{-1}\right)$ and $\mathrm{O}-\mathrm{H}\left(v_{\mathrm{O}-\mathrm{H}}\right.$ at $\left.3427 \mathrm{~cm}^{-1}\right){ }^{[17-19]}$. After reduction, the $\mathrm{O}-\mathrm{H}$ peak at $3427 \mathrm{~cm}^{-1}$ still can be observed, while the carboxyl peak at $1722 \mathrm{~cm}^{-1}$ is unobvious. 1631 $\mathrm{cm}^{-1}$ is corresponding to the bending vibration of $\mathrm{C}=\mathrm{C}$. $1631 \mathrm{~cm}^{-1}$ peak in $\mathrm{rGO}-\mathrm{m}$ is obviously stronger than that in $\mathrm{rGO}$, and $\mathrm{C}-\mathrm{O}-\mathrm{C}$ peak around $1044 \mathrm{~cm}^{-1}$ is adverse, and a new peak at $1405 \mathrm{~cm}^{-1}$ appeared in the spectrum of $\mathrm{rGO}-\mathrm{m}$, which is due to the $\mathrm{C}=\mathrm{C}$ stretching vibration in benzenoid rings, which further indicating that $\mathrm{rGO}-\mathrm{m}$ is reduced more completely under alkaline condition. The rGO-m shows the $\mathrm{N}-\mathrm{H}$ wagging vibration at $830 \mathrm{~cm}^{-1}$ of secondary amine group ${ }^{[20]}$, and two new peaks 1270 and $1127 \mathrm{~cm}^{-1}$ in the spectrum of $\mathrm{rGO}-\mathrm{m}$ are correponed to the stretching vibration of $\mathrm{C}-\mathrm{N}$ and $\mathrm{C}=\mathrm{N}$, respectively [21], demonstrating the amino functionalization result of rGO-m ${ }^{[48,49]}$. Compared with rGO-m, the new peak 1572 $\mathrm{cm}^{-1}$ in the spectra of $\mathrm{rGO}-\mathrm{m} / \mathrm{PANI}$ and $3 \mathrm{D}-\mathrm{rGO}-\mathrm{m} /$ PANI is attributed to the $\mathrm{C}=\mathrm{C}$ stretching vibration in the quinoid, and the stretching of $\mathrm{C}=\mathrm{N}$ at $1127 \mathrm{~cm}^{-1}$ is obviously intense and broad, which is due to the stretching vibration of $\mathrm{C}-\mathrm{N} .^{+}$in the polarized PANI structure, indicating the oxidation state existence in PANI ${ }^{[22]}$. 3D-rGO-m/PANI shows less absorption peaks of oxygencontaining functional group than $\mathrm{rGO}-\mathrm{m} / \mathrm{PANI}$ since the $\mathrm{KOH}$ activation at high temperature removed some of oxygen-containing functional group in rGO-m. All the above peaks observed in the spectrum of $3 \mathrm{D} \mathrm{rGO}-\mathrm{m} /$ PANI suggest that the PANI net-works are successfully decorated on graphene sheets. 


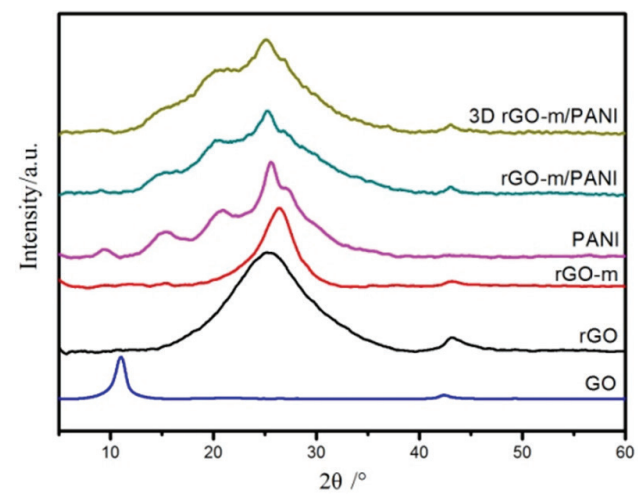

Figure 3. XRD patterns of GO, rGO, rGO-m, PANI, rGO-m/PANI and 3D rGO-m /PANI.

\subsection{Electrochemical performance}

Figure 5(a) shows the CV curves of the 3D rGO-m/PANI hybrid at a scan rate of 5, 10, 25, 50 and $100 \mathrm{mV} / \mathrm{s}$ in a 1 $\mathrm{M} \mathrm{H}_{2} \mathrm{SO}_{4}$ solution, respectively. 3D-rGO-m/PANI hybrid presents redox peaks, which are corresponding to the structural conversions of emeralddine/pernigraniline and leucoemeraldine/ emeraldine. These results indicate good pseudo- capacitance characteristics. The mirror-like
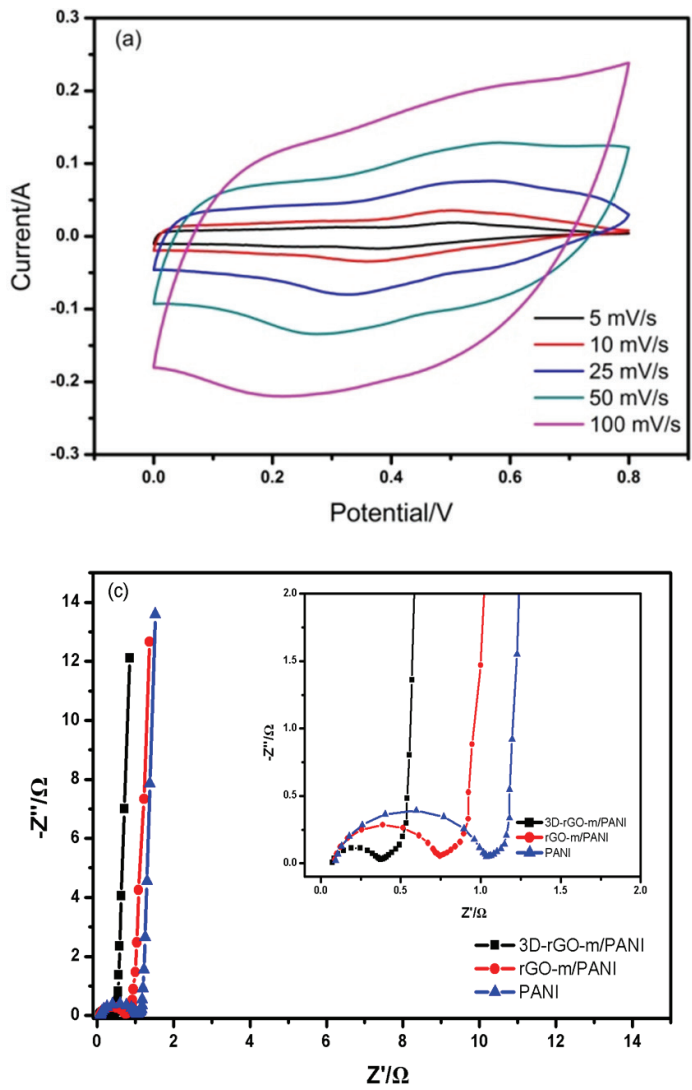

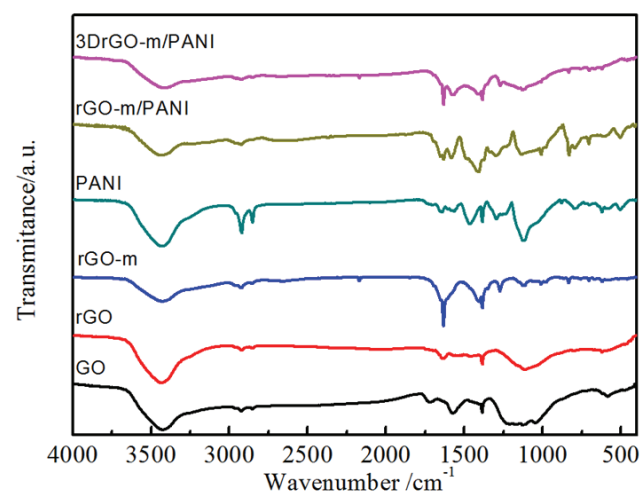

Figure 4. FT-IR spectra of GO, rGO, rGO-m, PANI, rGO-m/PANI and 3D rGO-m/PANI.

and symmetrical image in $0.5 \mathrm{~A} / \mathrm{g}$ galvanostatic charge -discharge (GCD) curves indicates that the reversible redox reaction proceed in 3D rGO-m/PANI, rGO-m/ PANI and PANI (figure 5(b)). The specific capacitance of 3D rGO-m/PANI calculated from GCD curves is $985 \mathrm{~F} / \mathrm{g}$ at $0.5 \mathrm{~A} / \mathrm{g}$, which is higher than that of $\mathrm{rGO}-\mathrm{m} / \mathrm{PANI}(633$ $\mathrm{F} / \mathrm{g})$ and PANI (405 F/g).
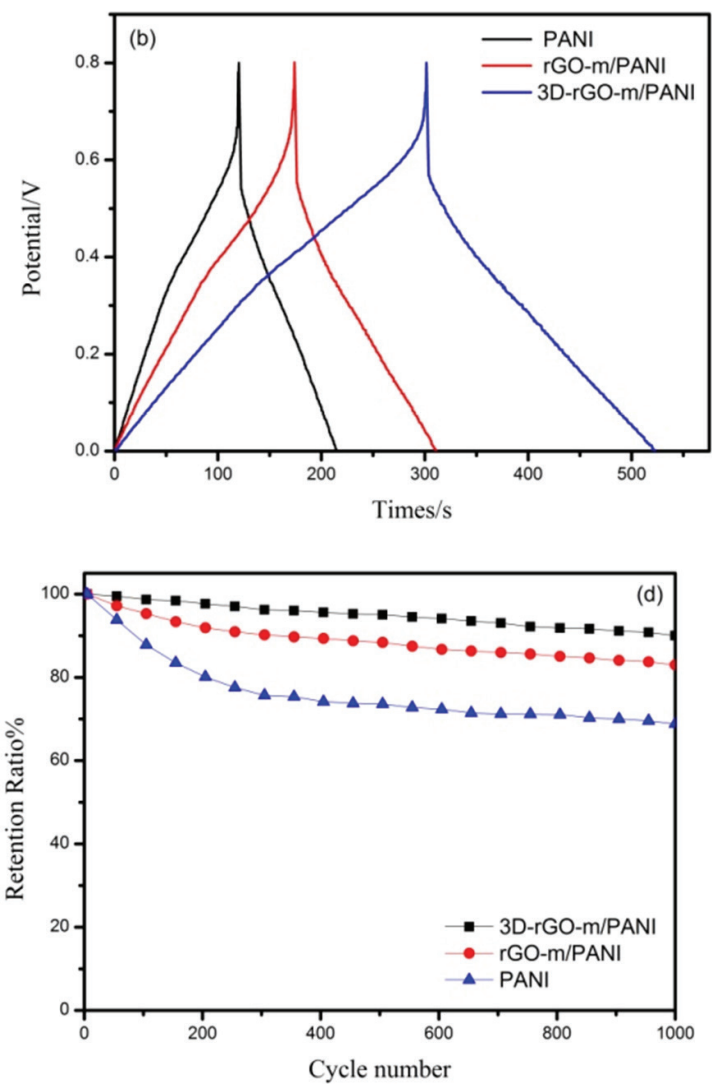

Figure 5. (a) CV curves of 3D rGO-m/PANI, (b) GCD curves at $0.5 \mathrm{~A} / \mathrm{g}$, (c) Nyquist plots and (d) cycling stability of 3D rGO-m/PANI, rGO-m/PANI and PANI.

Figure 5(c) depicts the Nyquist plots of 3D rGO-m / PANI and rGO-m/PANI hybrids. The obvious semicircle at the high frequency region can be seen. The straight line at the low frequency region is almost parallel to the vertical axis, which reflects the good capacity owing to the fast and reversible redox of PANI nanorod array. The first intersect 
of the Nyquist curve to the $Z^{\prime}$ axis represents equivalent series resistance $\left(R_{s}\right)$, and the diameter of the semicircle represents charge transfer resistance $\left(\mathrm{R}_{\mathrm{ct}}\right)$ at the electrode/ electrolyte interface. It can be seen that the 3D-rGO-m/ PANI has lowest $\mathrm{R}_{\mathrm{ct}}(0.3 \Omega)$ and $\mathrm{R}_{\mathrm{s}}(0.07 \Omega)$ than that of $\mathrm{rGO}-\mathrm{m} /$ PANI $\left(\mathrm{R}_{\mathrm{ct}}=0.7 \Omega, \mathrm{R}_{\mathrm{s}}=0.08 \Omega\right)$ and PANI $\left(\mathrm{R}_{\mathrm{ct}}=1.0\right.$ $\Omega, R_{s}=0.09 \Omega$ ). The low $R_{s}$ and $R_{c t}$ mean good electrical conductivity and fast charge transfer at the electrode and electrolyte interface, which can explain the high specific capacitance of 3D-rGO-m/PANI at a high current density. 3D rGO-m/PANI presents the energy density of 40.53 $\mathrm{Wh} / \mathrm{kg}$ at the power density of $0.66 \mathrm{~kW} / \mathrm{kg}$.

Figure $5(\mathrm{~d})$ is the cycling stability of the cell based on the 3D-rGO-m/PANI and rGO-m/PANI hybrids at 1 $\mathrm{A} / \mathrm{g}$ for 1000 cycles. The capacitance of 3D rGO-m/PANI after 1000 cycles still reaches $90 \%$ of its original one, while rGO-m/PANI only maitains $83 \%$ of its original one, the cycling stability of both hybrids are higher than that of pure PANI (69\%), so it is concluded that the cycling stability of 3D-rGO-m/PANI has been inhanced after $\mathrm{KOH}$ activation to $\mathrm{rGO}$. The improved cycling stability could be attributed to two aspects: (1) the three-dimensional crosslinking structure of 3D-rGO-m/PANI is favorable for the ion transport through a $3 \mathrm{D}$ continuous channel; (2) the electrode's volume and structure changes during the charge-discharge cycling are controlled to lower degree by $3 \mathrm{D}$ porous structure.

\section{Conclusions}

In summary, 3D cross-linking porous graphene was prepared by $\mathrm{KOH}$ activation to the 1,2,4-triaminobenzene modified graphene precursor (rGO-m). After in-situ chemical oxidative polymerization, 3D porous $\mathrm{rGO}-\mathrm{m} /$ PANI hybrids were obtained. The resulted $3 \mathrm{D}$ porous rGO-m/PANI hybrids possess PANI nanorod array on the $3 \mathrm{D}$ porous $\mathrm{rGO}-\mathrm{m}$. The $3 \mathrm{D}$ cross-linking porous structure provides high specific surface area, short and fast electrolyte diffusion pathways. The ion-diffusion and charge-transfer resistances of the 3D porous rGO-m/ PANI hybrids are decreased. The specific capacitance is enhanced markedly compared to inactivated rGO-m/ PANI hybrids and pure PANI. The maximum specific capacitance is $985 \mathrm{~F} / \mathrm{g}$ at $0.5 \mathrm{~A} / \mathrm{g}$. Therefore, 3D porous rGO-m/PANI hybrids maybe become promising electrode materials for application in supercapacitors.

Acknowledgements: This work was financially supported by the Program of National Natural Science Foundation of China (No. 51472166) and Liaoning BaiQianWan Talents program.

\section{References}

[1] Zhang L L and Zhao X S. Carbon-based materials as supercapacitor electrodes[J]. Chem. Soc. Rev., 2009, 38(9):2520-2531

[2] Chen S, Xing W, Duan J J, et al. Nanostructured morphology control for efficient supercapacitor
electrodes[J]. J Mater. Chem. A, 2013,1:2941-2954

[3] Stoller MD, Park S, Zhu Y, et al. Graphene-based ultracapacitors[J]. Nano Lett., 2008, 8(10):3498-3502

[4] Zhao B, Liu P, Jiang $Y$, et al. Supercapacitor performances of thermally reduced graphene oxide[J]. J Power Sources, 2012,198: 423-427

[5] Li L, Raji A R, Fei H, et al. Graphene oxide-based benzimidazole-crosslinked networks for highperformance supercapacitors[J]. ACS Appl. Mater. Interfaces, 2013, 5(18): 8367-8374

[6] Ćirić-Marjanović G. Recent advances in polyaniline research: polymerization mechanisms, structural aspects, properties and applications[J]. Synth Met., 2013, 177: 1-47

[7] Liu Y, Ma Y, Guang S Y, et al. Facil fabrication of threedimentional highly ordered structural polyanilinegraphene bulk hybrid materials for high performance supercapacitor electrodes[J]. J Mater. Chem. A, 2014, 2(3): 813-823

[8] Zhao X, Gnanaseelan M, Jehnichen D, et al. Green and facile synthesis of polyaniline/tannic acid/rGO composites for supercapacitor purpose[J]. Journal of Materials Science, 2019, 54(15):10809-10824.

[9] Hu R, Zhao J, Zhu G, et al. Fabrication of flexible free-standing reduced graphene oxide/polyaniline nanocomposite film for all-solid-state flexible supercapacitor[J]. Electrochimica Acta, 2018, 261:151159.

[10] Wang X, Zhao J, Li Z, et al. Effects of preparation conditions on the supercapacitor performances of $\mathrm{MnO2-PANI/titanium} \mathrm{foam} \mathrm{composite} \mathrm{electrodes[J].}$ Journal of Nanoparticle Research, 2019, 21(6):119-133.

[11] Kaushal I, Sharma A K, Saharan P, et al. Superior architecture and electrochemical performance of $\mathrm{MnO} 2$ doped PANI/CNT graphene fastened composite[J]. Journal of Porous Materials, 2019, 26(5):1287-1296.

[12] Lu X, Li L, Song B, et al. Mechanistic investigation of the graphene functionalization using $\mathrm{p}$-phenylenediamine and its application for supercapacitors[J]. Nano Energy, 2015, 17: 160-170

[13] Li L Y, Song B, Maurer L, et al. Molecular engineering of aromatic amine spacers for high-performance graphene-based supercapacitors[J]. Nano Energy, 2016, 21:276-294

[14] Kumar N A, Baek J B. Polyaniline-grafted reduced graphene oxide for efficient electrochemical supercapacitors[J]. ACS Nano, 2012, 6(2): 1715-1723

[15] Yan J, Wei T, Fan Z J, et al. Preparation of graphene nanosheet/carbon nanotube/polyaniline composite as electrode material for supercapacitors[J]. J Power Sources, 2010, 195(9): 3041-3045

[16] Yan J, Wei T, Shao B, et al. Preparation of a graphene nanosheet/polyaniline composite with high specific 
capacitance[J]. Carbon, 2010, 48(2): 487-493

[17] Raghu A V, Lee Y R, Jeong $H$ M, et al. Preparation and physical properties of waterborne polyurethane/ functionalized graphene sheet nanocomposites[J]. Macromolecular Chemistry and Physics, 2008, 209(24): 2487-2493

[18] Shin H J, Kim J, Benayad A, et al. Efficient reduction of graphite oxide by sodium borohydride and its effect on electrical conductance[J]. Advanced Functional Materials, 2009, 19(12): 1987-1992

[19] Titelman G, Gelman V, Bron S, et al. Characteristics and microstructure of aqueous colloidal dispersions of graphite oxide[J]. Carbon, 2005, 43(3): 641-649
[20] Liu Y, Ma Y, Guang S Y, et al. Polyaniline-graphene composites with a three-dimentional array-based nanostructure for high performance supercapacitors[J]. Carbon, 2015, 83: 79-89

[21] Li G C, Jiang L, Peng H R. One-dimensional polyaniline nanostructures with controllable surfaces and diameters using vanadic acid as the oxidant[J]. Macromolecules, 2007, 40(22): 7890-7894

[22] $\mathrm{Hu} \mathrm{H}$, Liu S W, Hanif $M$, et al. Three-dimentional cross-lingked carbon network wrapped with ordered polyaniline nanowires for high-performance pseudosupercapacitors[J]. Journal of power sources, 2014, 268: 451-458 